\title{
A new method to estimate the sol-gel transition entropy in physically gelling systems
}

\author{
Takashi Sakai, Jun-ichi Horinaka and Toshikazu Takigawa
}

\begin{abstract}
A Clapeyron-type equation is presented to estimate the transition entropy for the sol-gel transition of physical gels, based on simple thermodynamics for the gels under load. The transition entropy and the transition enthalpy were estimated for $\kappa$-carrageenan/water and gellan/water systems using this Clapeyron-type equation. For the carrageen systems, the transition entropy per unit gel volume $\left(\Delta S_{V}\right)$ remained constant, regardless of the polymer concentration and the species and concentration of the added salts. A similar behavior was also observed for the gellan systems. These results suggest that the structure of the crosslink domains of the gels is almost identical, regardless of the polymer concentration and the species and concentration of the added salts, if the comparison was made with a fixed polysaccharide. The values of $\Delta S_{V}$ obtained in this study were higher than those obtained using calorimetry as reported in the literature; however, the difference was not large.

Polymer Journal (2015) 47, 244-248; doi:10.1038/pj.2014.98; published online 5 November 2014
\end{abstract}

\section{INTRODUCTION}

Polymer gels can be divided into two groups: chemical gels, in which the crosslinks are formed through covalent bonds, and physical gels, in which the crosslinks are formed through non-covalent bonds such as a series of hydrogen bonds and microcrystal domains. ${ }^{1,2}$ Solutions of some polymers form physical gels upon cooling if the polymer concentration $(c)$ is higher than a threshold. In this cooling process, the 'freezing point' at which the system loses its fluidity is called the sol-gel transition temperature $\left(T_{\text {tr }}\right)$ upon cooling. Physical gels melt at a certain temperature upon heating and recover the solution state (or sol state). This melting temperature corresponds to the $T_{\text {tr }}$ upon heating. Generally, because these two $T_{\text {tr }}$ values are different, a large hysteresis is observed for the sol-gel transition of physical gels. ${ }^{3,4}$

Many studies have clarified the sol-gel transition behavior of physical gels. ${ }^{1-4}$ Among the physical gels, much attention has been paid to hydrogels made of polysaccharide in the food industry, and the thermal and rheological properties of various polysaccharide systems have been examined. ${ }^{2-4}$ The gelation of polysaccharide/water systems occurs owing to the aggregation of helical domains (single-helix or double-helices) followed by the coil-to-helix transition of the polymer chains. ${ }^{5-8}$ In this case, both the transition enthalpy $(\Delta H)$ and $T_{\text {tr }}$ are related to the dimension and/or structure of the crosslink domains. The aggregation after the helix-to-coil transition is considered to be a type of 'freezing'. Therefore, the sol-gel transition of polysaccharide gels is also owing to the melting/freezing of crosslink domains. The transition temperature $T_{\mathrm{tr}}$ can be easily determined using several methods. Dynamic-viscoelasticity measurements upon heating (or cooling) are now widely used for this determination. ${ }^{7}$ Available methods for the estimation of $\Delta H$ appears to be limited; however, micro-differential scanning calorimetry (micro-DSC) is often used for the estimation. ${ }^{8-10}$ Micro-DSC detects the thermodynamic transition even in a dilute regime where the sol-transition never occurs and instead a sol-sol transition (only a conformational transition such as the helix-coil transition, for example) takes place. Therefore, the $\Delta H$ determined using micro-DSC is not purely because of the sol-gel transition but due to the melting/freezing transition of the crosslink domains.

Concerning the sol-gel transition itself, we implicitly know that the entropy $(S)$ may show a discontinuity at the transition point because the translational entropy should disappear by gelation; ${ }^{1}$ however, little is known about the real behavior of the derivatives of the free energy. ${ }^{11}$ The 'coexistence' of two phases at the transition point is key to understanding the phase equilibrium; however, this coexistance may also cause difficulty in the case of the sol-gel transition because the concept of the coexistence is basically incompatible with the sol-gel transition. Thus, there still remains uncertainty in this type of transition. This uncertainty may imply that the sol-gel transition itself might be regarded as a geometrical transition such as a percolation; $;^{1,12}$ however, it is still worth searching for a method to provide the transition entropy (or enthalpy) that originates purely from the sol-gel transition. For this purpose, the introduction of mechanical work to the gels is attempted to increase the variance degree of freedom. The aim of this study is twofold: one is to formulate an equation to provide the entropy change at the transition for physical gels under load, as in the case of the volume phase transition of polymer gels, ${ }^{13,14}$ and the second is to apply the above equation to $\kappa$-carrageenan/water and gellan/water systems to verify the applicability of the proposed method. 


\section{THEORETICAL APPROACH}

Here, we focus on the sol-gel transition of physical gels under load. Let $G(T, f)$ be the free energy of the gels defined by a Legendre transform of the internal energy $(U)$ as

$$
G(T, \boldsymbol{f})=U(S, \boldsymbol{L})-T S-\boldsymbol{f} \cdot \boldsymbol{L}
$$

where $T$ is the temperature, $f$ is the external (uniaxial) force and $L$ is the displacement vector. The work by the pressure is assumed to be negligible and therefore the infinitesimal change of $U(\mathrm{~d} U)$ is given by $\mathrm{d} U=T \mathrm{~d} S+f \cdot \mathrm{d} \boldsymbol{L}$. If the gel under load (force $\boldsymbol{f}$ ) 'coexists' with the sol at $T$, then the gel and the sol must satisfy the equilibrium condition:

$$
G_{\text {gel }}(T, \boldsymbol{f})=G_{\text {sol }}(T, \boldsymbol{f})
$$

where $G_{\text {gel }}(T, f)$ and $G_{\text {sol }}(T, f)$ are the $G(T, f)$ of the gel and the sol, respectively. In the sol phase, $f$ vanishes at equilibrium because the sol is a liquid; therefore, $G_{\text {sol }}(T, f)$ can be written as $G_{\text {sol }}(T, f)=G_{\text {sol }}(T)$, which simplifies the equilibrium condition to

$$
G_{\text {gel }}(T, f)=G_{\text {sol }}(T)
$$

If the coexistence is also assumed at another point $(T+\mathrm{d} T, \boldsymbol{f}+\mathrm{d} f)$, then the equilibrium condition at this point is given by

$$
G_{\text {gel }}(T+\mathrm{d} T, \boldsymbol{f}+\mathrm{d} \boldsymbol{f})=G_{\text {sol }}(T+\mathrm{d} T)
$$

An equation determining the slope of the coexistence curve (or phase boundary) is obtained from equations (3) and (4) as

$$
\Delta S \mathrm{~d} T+\boldsymbol{L}_{g e l} \cdot \mathrm{d} \boldsymbol{f}=0
$$

where $\Delta S=\left(S_{\text {gel }}-S_{\text {sol }}\right)$ with $S_{\text {gel }}=\left(\partial G_{\text {gel }} / \partial T\right)_{f}, S_{\text {sol }}=\mathrm{d} G_{\text {sol }} / \mathrm{d} T$, and $L_{\mathrm{gel}}=\left(\partial G_{\mathrm{gel}} / \partial f\right)_{T}$. Assuming that the shape of the gel is cylindrical and $\boldsymbol{f}$ acts downwards on the circular area of the cylinder, we can rewrite equation (5) as

$$
-\frac{\Delta S}{V_{\mathrm{tr}}} \mathrm{d} T=\frac{L_{\mathrm{gel}}}{h_{0}-L_{\mathrm{gel}}} \frac{\mathrm{d} f}{s_{\mathrm{tr}}}=\frac{h_{0}-h_{\mathrm{tr}}}{h_{\mathrm{tr}}} \frac{\mathrm{d} f}{s_{\mathrm{tr}}}
$$

where $L_{\text {gel }}$ and $d f$ are defined by $L_{\text {gel }}=\left|L_{\text {gel }}\right|$ and $\mathrm{d} f=|\mathrm{d} f|$, respectively. The quantities $h_{0}$ and $h_{\mathrm{tr}}$ are the initial height of the gel and the height at the transition temperature, respectively. $V_{\mathrm{tr}}$ and $s_{\mathrm{tr}}$ are the volume and the force-acting area at the transition temperature, respectively. For $h_{\mathrm{tr}}$, we have $h_{\mathrm{tr}}=h_{0}-L_{\mathrm{gel}}$ and we also have $V_{\mathrm{tr}}=h_{\mathrm{tr}} s_{\mathrm{tr}}$ for $V_{\mathrm{tr}}$. Equation (6) can be re-written as

$$
-\Delta S_{V}=\varepsilon\left(\frac{\mathrm{d} \widetilde{\sigma}}{\mathrm{d} T}\right)_{\text {coex }}
$$

where $\mathrm{d} \widetilde{\sigma}$ is given by $\mathrm{d} \widetilde{\sigma}=\mathrm{d} f / s_{\text {tr }}$ and $\varepsilon$ is defined by

$$
\varepsilon=\frac{h_{0}-h_{\mathrm{tr}}}{h_{\mathrm{tr}}}
$$

The quantity $\varepsilon$ is the critical strain for the transition and $\widetilde{\sigma}$ is the true stress. The subscript 'coex' in equation (7) stands for the coexistence curve. The quantity $\Delta S_{\mathrm{V}}$ in equation (7) is defined by $\Delta S_{\mathrm{V}}=\Delta S / V_{\mathrm{tr}}$ and corresponds to the transition entropy per unit volume. Equation (7) states that the change in entropy at the sol-gel transition point, which we call the transition entropy, is determined by $T_{\text {tr }}, \varepsilon$ and the slope of the coexistence curve $(\mathrm{d} \widetilde{\sigma} / \mathrm{d} T)_{\text {coex }}$; this equation is quite similar to the Clapeyron equation. Because we can expect the relationship $\Delta S_{\mathrm{V}}=\Delta H_{\mathrm{V}} / T_{\mathrm{tr}}$, equation (7) can also be converted to the following expression with the transition enthalpy per unit volume $\left(\Delta H_{\mathrm{V}}\right)$

$$
-\frac{\Delta H_{V}}{T_{\mathrm{tr}}}=\varepsilon\left(\frac{\mathrm{d} \tilde{\sigma}}{\mathrm{d} T}\right)_{\text {coex }}
$$

\section{EXPERIMENTAL PROCEDURE}

Powdery $\mathrm{K}$-carrageenan and gellan were purchased from Tokyo Chemical Industry (Tokyo, Japan), and Wako (Osaka, Japan), respectively, and both were used without further purification. Table 1 summarizes the metal content of the polysaccharides analyzed using ICP-AES (inductively coupled plasma-atomic emission spectroscopy). The polysaccharide hydrogels were prepared by using pure water or salt solutions as solvents. The salts used were $\mathrm{NaCl}$ and $\mathrm{KCl}$, which were purchased from Wako. First, the polysaccharide solutions were prepared at $80^{\circ} \mathrm{C}$ and poured into a mold to obtain cylindrical samples. Gelation was performed by keeping the solutions at $5^{\circ} \mathrm{C}$ for $12-18 \mathrm{~h}$. After removing the gels from the mold, the cylindrical samples were maintained in the solvent at $25^{\circ} \mathrm{C}$ for $\sim 20 \mathrm{~min}$ for equilibration, and then initial diameter $\left(d_{0}\right)$ and initial height $\left(h_{0}\right)$ of the samples were measured. Table 2 summarizes the sample-code, polymer concentration $(c)$, concentration of added salt $\left(c_{\mathrm{s}}\right)$, initial diameter $\left(d_{0}\right)$ and initial height $\left(h_{0}\right)$ of the samples. The sample code is composed of two blocks: the left block indicates the type of polysaccharide used (C; carragenan: G; Gellan) and the value of ten times the polymer concentration, and the right block indicates the type of salt used in the solvent $(\mathrm{Na} ; \mathrm{NaCl}$ : $\mathrm{K} ; \mathrm{KCl}$ ) and the salt concentration in mM. A blank in the right block indicates that the pure water was used as the solvent.

The cylindrical sample was set in a transparent rectangular container that was filled with the same solvent as used for the sample preparation. A plastic (acrylic glass) plate was mounted on the upper side of the gel, and then metal weights were added on the plate. The compression force $(f)$ acting on the gel was controlled by the metal weights, and a correction by buoyancy was made for the plate and the weights. The transparent container was then settled in a water bath. The temperature of the gel and the surrounding solvent in the container was finely controlled by controlling the water temperature in the bath. The temperature of the gel and the solvent was raised stepwise by $1{ }^{\circ} \mathrm{C}$, and the typical heating rate was approximately $1{ }^{\circ} \mathrm{C}$ per $10 \mathrm{~min}$. Preliminary tests were performed to obtain a rough estimate of $T_{\text {tr }}$, and then the experiments proceeded at a temperature lower than the preliminary $T_{\text {tr }}$ by $\sim 3-4{ }^{\circ} \mathrm{C}$. The transition temperature $T_{\text {tr }}$ was defined as $T_{\text {tr }}=T_{\max }+(1 / 2)$, where

Table 1 The metal ion content of the polysaccharides analyzed using ICP-AES

\begin{tabular}{lcccc}
\hline & $\mathrm{Na}^{+} / \mathrm{wt} \%$ & $\mathrm{~K}^{+} / \mathrm{wt} \%$ & $\mathrm{Ca}^{2+/} \mathrm{wt} \%$ & $\mathrm{Mg}^{2+/} \mathrm{wt} \%$ \\
\hline $\boldsymbol{\kappa}$-carrageenan & 2.00 & 4.8 & 0.35 & - \\
Gellan & 0.46 & 3.6 & 0.31 & 0.089 \\
\hline
\end{tabular}

Table 2 The sample code, polymer concentration (c), concentration of added salt $\left(c_{\mathrm{s}}\right)$, initial diameter $\left(d_{0}\right)$ and initial height $\left(h_{0}\right)$ of the samples

\begin{tabular}{lcccc}
\hline Sample code & $c / w t \%$ & $c_{s} / m M$ & $d_{0} / \mathrm{mm}$ & $h_{0} / \mathrm{mm}$ \\
\hline C15 & 1.5 & - & 10.03 & 10.08 \\
C20 & 2.0 & - & 10.03 & 10.08 \\
C15-Na25 & 1.5 & 25 & 10.13 & 10.08 \\
C15-Na50 & 1.5 & 50 & 10.08 & 10.10 \\
C20-Na25 & 2.0 & 25 & 10.10 & 10.08 \\
C15-K25 & 1.5 & 25 & 10.03 & 10.05 \\
C15-K50 & 1.5 & 50 & 10.03 & 10.03 \\
C20-K25 & 2.0 & 25 & 10.07 & 10.07 \\
G15 & 1.5 & - & 10.30 & 10.08 \\
G20 & 2.0 & - & 10.27 & 10.10 \\
G10-Na25 & 1.0 & 25 & 10.18 & 10.10 \\
G10-Na50 & 1.0 & 50 & 10.10 & 10.07 \\
G15-Na25 & 1.5 & 25 & 10.12 & 10.08 \\
G10-K25 & 1.0 & 25 & 10.20 & 10.10 \\
G10-K35 & 1.0 & 35 & 10.37 & 10.10 \\
\hline
\end{tabular}


$T_{\max }$ is the maximum (highest) survival temperature of the gels upon heating at $1{ }^{\circ} \mathrm{C}$ intervals. The height of the gel at $T, h(T)$ was monitored during heating using a CCD (charged coupled device) camera with a DVD (digital versatile disc) recorder. Because $\tilde{\sigma}$ defined in the previous section is the true stress, experimental values of $\widetilde{\sigma}$ were calculated by $\widetilde{\sigma}=f / s_{\text {tr }}$ with the assumptions $s_{\text {tr }}=$ $s_{0} h_{0} / h_{\mathrm{tr}}$. Here, $s_{0}$ is the initial force-acting area given by $s_{0}=\pi d_{0}^{2} / 4$, and $h_{\mathrm{tr}}$ was estimated by extrapolation using $h\left(T_{\max }-1\right)$ and $h\left(T_{\max }\right)$.

\section{RESULTS AND DISCUSSION}

Figure 1 shows $h$ plotted against $T$ for the $\kappa$-carrageenan hydrogel C15. At a constant $f$, as traced by a solid line, $h$ monotonically decreases with increasing $T$ and finally reaches the point $h=0$, where the hydrogel sample melted completely and spread out in the bath. By setting this temperature as $\left(T_{\max }+1\right)$, the experimental transition temperature $T_{\mathrm{tr}}$ is defined as $T_{\mathrm{tr}}=T_{\max }+(1 / 2)$ because the temperature was controlled in an interval of $1{ }^{\circ} \mathrm{C}$. The determined points of $T_{\text {tr }}$ for various forces are shown by filled symbols in the figure, indicating that $T_{\text {tr }}$ decreases with increasing $f$. This behavior is also observed for the other $\kappa$-carrageenan gel samples. In Figure 2, the $h$ vs $T$ plots of the gellan hydrogel G15 are shown. The value of $c$ for this sample is $1.5 \mathrm{wt} \%$, which is identical to that of the gellan gel shown in Figure 1. As is the case of $\mathrm{C} 15$, the $T_{\operatorname{tr}}$ of this ample also decreases with increasing $f$; however, the $T_{\text {tr }}$ for gellan is much higher than that for $\kappa$ carrageenan (more than $10^{\circ} \mathrm{C}$ higher).

Figure 3 shows the $\widetilde{\sigma}$ dependence of $T_{\text {tr }}$ for the $\kappa$-carrageenan gels. For all the gel samples, the $T_{\text {tr }}$ linearly decreases with increasing $\widetilde{\sigma}$, and the slope appears to be almost identical for all the gel samples. As seen from equation (7) and equation (9), this slope corresponds to

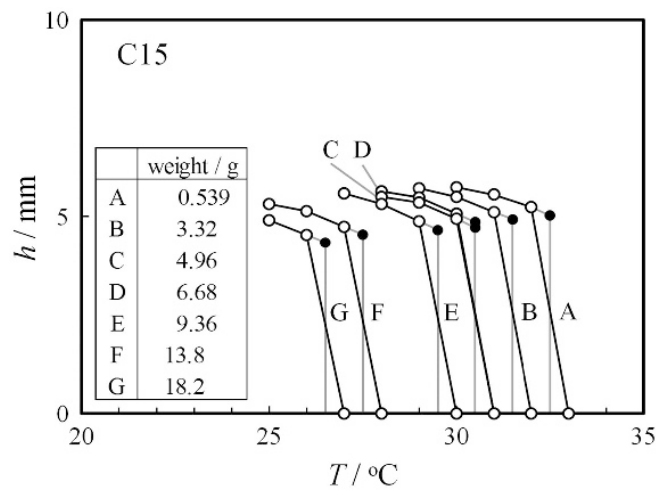

Figure $1 T$ dependence of $h$ for C15 under load. Symbols A-G differ in applied weight.

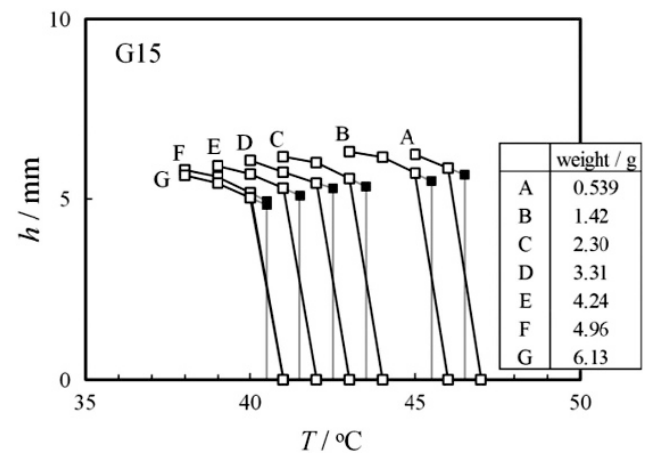

Figure $2 T$ dependence of $h$ for G15 under load. Symbols A-G differ in applied weight. $(\partial T / \partial \widetilde{\sigma})_{\text {coex }}$. Similar plots for the gellan gels are shown in Figure 4. The vertical axis differs in magnification between panel (a) and panel (b); however, the slope becomes identical for all of the samples. This slope value is slightly higher compared with that for the $\kappa$-carrageenan gels, although we do not know the reason at present. It should be recalled that each line in Figures 3 and 4 also becomes a type of phase boundary of the corresponding gel sample. The sol phase is the region above the line, whereas the gel phase is the region below the line. In Figure 5, $\varepsilon$ is plotted against $\widetilde{\sigma}$ for $\mathrm{C} 15$ and G15 as examples. Because $\varepsilon$ expresses the ratio of the compressed length to the retained one (see equation (8)) it is reasonable that $\varepsilon$ increases with increasing $\widetilde{\sigma}$ for both gels. The non-zero values of the intercept originate from the deformation of gels owing to their own weight; however, the difference

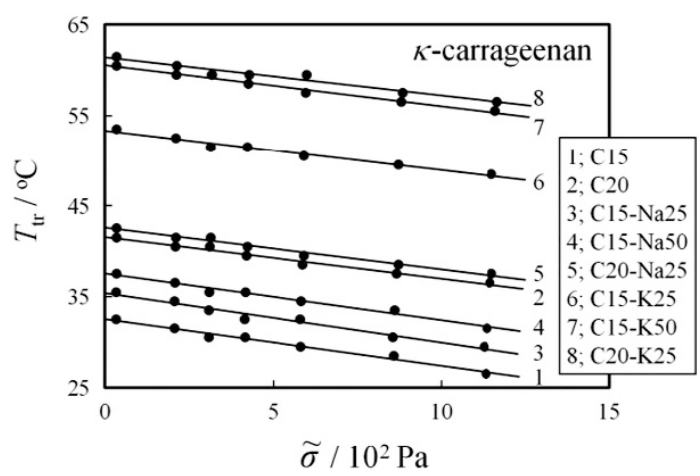

Figure $3 \tilde{\sigma}$ dependence of $T_{\text {tr }}$ for the $\kappa$-carrageenan gels.
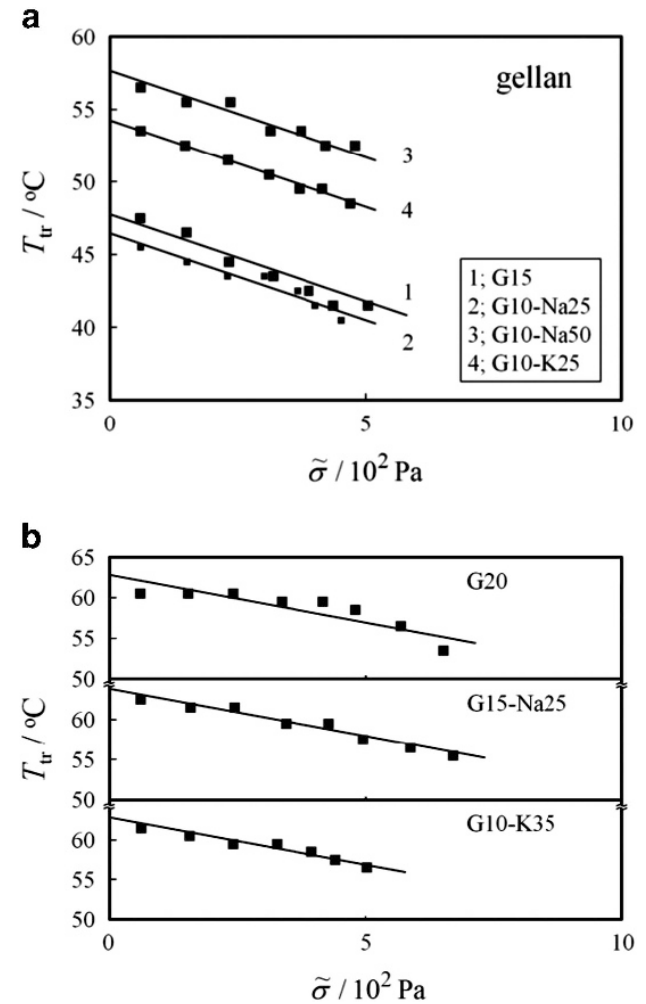

Figure $4 \widetilde{\sigma}$ dependence of $T_{\text {tr }}$ for the gellan gels: (a) plots for four samples with lower $T_{\mathrm{tr}}$. The small filled symbol represents G10-K25; (b) separate plots for three samples with higher and lower $T_{\mathrm{tr}}$. 


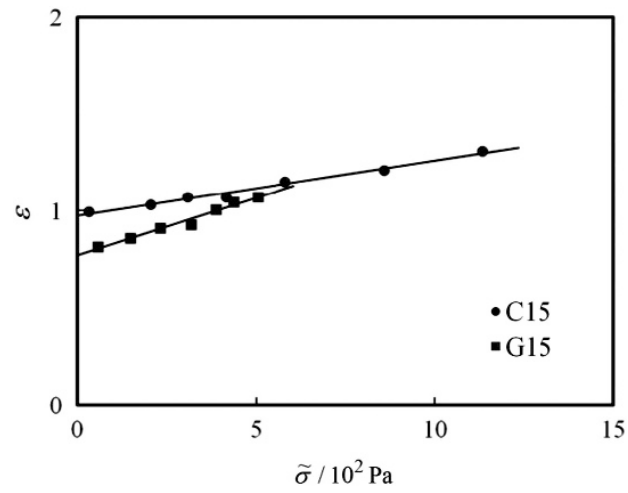

Figure $5 \widetilde{\sigma}$ dependence of $\varepsilon$ for $\mathrm{C} 15$ and G15.

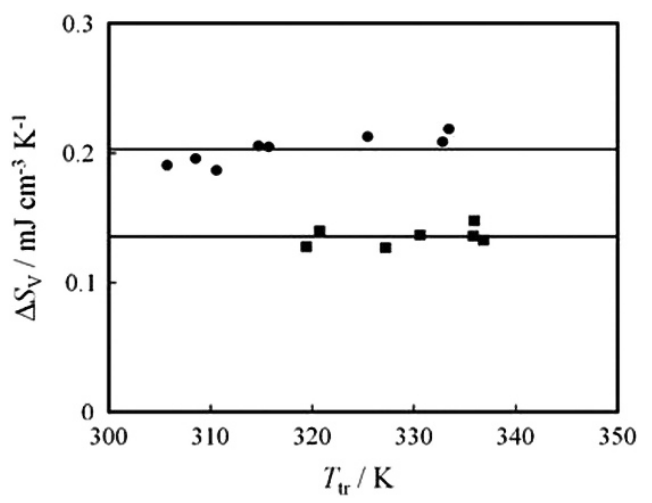

Figure 6 Normalized transition entropy $\Delta S_{\mathrm{V}}$ plotted against $T_{\mathrm{tr}}$ for the $\kappa$ carrageenan gels (circles) and the gellan gels (squares).

in these values is not large. The higher slope for gellan reflects the fact that the gellan gel is softer than the carrageenan gel.

Although, $\Delta S_{\mathrm{V}}$ defined in this paper depends on force as well as temperature, here we use the limiting values of $\Delta S_{\mathrm{V}}$ at $\widetilde{\sigma} \rightarrow 0$. This limit can be easily made by using $\varepsilon$ at $\widetilde{\sigma} \rightarrow 0$ and the constant value of $(\partial T / \partial \widetilde{\sigma})_{\text {coex }}$. In Figure $6, \Delta S_{\mathrm{V}}$ at $\widetilde{\sigma} \rightarrow 0$ is plotted against $T_{\text {tr }}$ for the $\kappa-$ carrageenan gels and the gellan gels. The data points for each of the gels remain constant, independently of $T_{\text {tr. }}$. The upper line represents the average values for the carrageenan gels (average $\Delta S_{\mathrm{V}}=0.20 \mathrm{~mJ}$ $\mathrm{cm}^{-3} \mathrm{~K}^{-1}$ ), and the lower line corresponds to the average of the gellan gels (average $\Delta S_{\mathrm{V}}=0.14 \mathrm{~mJ} \mathrm{~cm}^{-3} \mathrm{~K}^{-1}$ ). The difference in the $\Delta S_{\mathrm{V}}$ between the two groups mainly originates from the difference in $(\partial T / \partial \widetilde{\sigma})_{\text {coex }}$. As previously stated, the structure of the crosslink domains is still controversial for polysaccharide gels; however, aggregates of double-helices are widely considered to function as crosslinks. ${ }^{5,8}$ The fact that $\Delta S_{\mathrm{V}}$ remains constant in an individual group suggests that the structure of the aggregates prior to melting is almost identical, regardless of polymer concentration, as well as, the species and concentration of added salts if the polysaccharide species is fixed. However, the different polysaccharide systems provide different $\Delta S_{\mathrm{V}}$ values, which indicates that there exists no regularity such as a Richard's rule governing the physical gels. In Figure $7, \Delta H_{\mathrm{V}}$ converted from $\Delta S_{\mathrm{V}}$ and $T_{\mathrm{tr}}$ is re-plotted against $T_{\mathrm{tr}}$. Here, each line corresponds to the average of $\Delta S_{\mathrm{V}}$ in the previous figure. Interestingly, the $\kappa$ carrageenan group shows a higher $\Delta H_{\mathrm{V}}$ than the gellan group when compared at the same $T_{\mathrm{tr}}$. The numerical values of $T_{\mathrm{tr}}, \Delta S_{\mathrm{V}}$ and $\Delta H_{\mathrm{V}}$

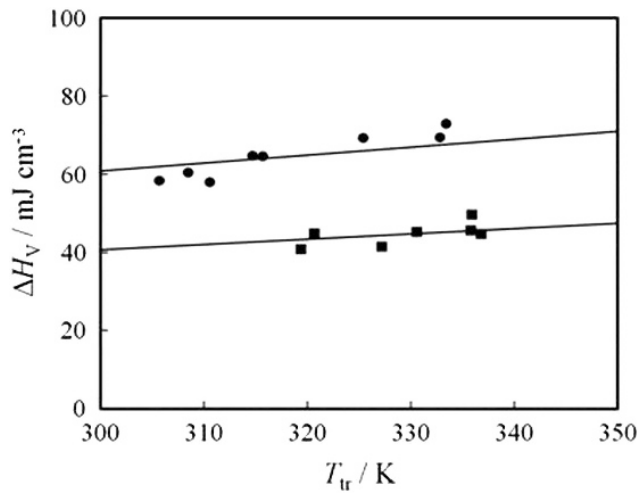

Figure 7 Normalized transition enthalpy $\Delta H_{v}$ plotted against $T_{\text {tr }}$ for the $\kappa$-carrageenan gels (circles) and the gellan gels (squares).

Table $3 T_{\text {tr }}, \Delta S_{\mathrm{V}}$ and $\Delta H_{\mathrm{V}}$ for the $\kappa$-carrageenan and gellan gels

\begin{tabular}{lccc}
\hline Sample & $T_{\text {tr }} / \mathrm{K}$ & $\Delta \mathrm{S}_{V} / \mathrm{mJ}$ & $\Delta \mathrm{H}_{V} / \mathrm{mJ} \mathrm{Cm}^{-3}$ \\
\hline C15 & 305.7 & 0.19 & 58 \\
C20 & 314.7 & 0.21 & 65 \\
C15-Na25 & 308.5 & 0.20 & 60 \\
C15-Na50 & 310.6 & 0.19 & 58 \\
C20-Na25 & 315.7 & 0.21 & 65 \\
C15-K25 & 325.4 & 0.21 & 69 \\
C15-K50 & 332.8 & 0.21 & 70 \\
C20-K25 & 333.4 & 0.22 & 73 \\
G15 & 321.4 & 0.14 & 45 \\
G20 & 336.3 & 0.15 & 50 \\
G10-Na25 & 318.5 & 0.13 & 41 \\
G10-Na50 & 331.3 & 0.14 & 45 \\
G15-Na25 & 336.7 & 0.13 & 45 \\
G10-K25 & 327.4 & 0.13 & 42 \\
G10-K35 & 335.4 & 0.14 & 46 \\
\hline
\end{tabular}

for the polysaccharide gels obtained in this study are summarized in Table 3.

Oakenfull et al. ${ }^{9}$ have reported the transition temperature and the transition enthalpy for the sol-gel transition of $k$-carrageenan systems. Using their data for a $0.5 \mathrm{wt} \%$ system, we can report $\Delta S_{\mathrm{V}}=0.16$ $\mathrm{mJ} \mathrm{cm}{ }^{-3} \mathrm{~K}^{-1}$. Del Carmen Núñez-Santiago and Tecante have also reported that $\Delta S_{\mathrm{V}}=0.11 \mathrm{~mJ} \mathrm{~cm}^{-3} \mathrm{~K}^{-1}$ if we use the data for a $0.5 \mathrm{wt}$ $\%$ system. Both values are lower than the average $\Delta S_{\mathrm{V}}$ estimated in this study. Miyoshi and Nishinari ${ }^{7}$ have reported similar data for a potassium-type gellan in salt solutions. Converting their values for the $1.0 \mathrm{wt} \%$ system into $\Delta S_{\mathrm{V}}$ we have $0.076 \mathrm{~mJ} \mathrm{~cm}^{-3} \mathrm{~K}^{-1}$ for the gellan gel. Thus, the $\Delta S_{\mathrm{V}}$ obtained in this study is high compared with those obtained using calorimetry; however, the difference is not large despite the differences in the sample characteristics. This difference might be expected because $\Delta S_{\mathrm{V}}$ by micro-DSC reflects only the heat of fusion of the helical domains. This should be clarified by further studies; however, the method presented here is useful for the estimation of the transition entropy (and enthalpy) of physical gels.

\section{CONCLUSIONS}

A Clapeyron-type equation for the sol-gel transition of physical gels was formulated, and the transition entropy, as well as, the transition enthalpy was also estimated for $\kappa$-carrageenan/water and gellan/water systems based on this equation. For the carrageen gels, the $\Delta S_{\mathrm{V}}$ 
remained constant regardless of polymer concentration, as well as, the species and concentration of added salts. A similar behavior was observed for the gellan gels. These results suggest that the structure of the crosslink domains of the gels is almost identical, regardless of polymer concentration and the species and concentration of added salts for a fixed polysaccharide. However, because the $\Delta S_{V}$ depended on the type of polysaccharide, there existed no regularity such as a Richard's rule for the polysaccharide gels. The value of $\Delta S_{\mathrm{V}}$ obtained in this study was higher than those obtained using calorimetry; however, the difference was not large. We have shown that the Clapeyron-type equation presented here is useful for the estimation of the sol-gel transition entropy (and enthalpy) of physical gels.

1 de Gennes, P. G. Scaling Concept in Polymer Physics. 222-246 (Cornell University Press: Ithaca, 1979).

2 Ross-Murphy, S. B. Physical gelation of synthetic and biological macromolecules in Polymer Gels (eds DeRossi D., Kajiwara K., Osada Y., Yamauchi A.) 21-39 (Plenum Press: New York, 1991).
3 Clark, A. H. \& Ross-Murphy, S. B. Structural and mechanical properties of biopolymer gels. Adv. Polym. Sci. 83, 57-192 (1987).

4 te Nijenhuis, K. Thermoreversible networks. Adv. Polym. Sci. 130, 1-252 (1997).

5 Rees, D. A. Polysaccharide shapes and their interactions-some recent advances. Pure Appl. Chem. 53, 1-14 (1981).

6 Smidsrød O. \& Grasdalen H. Some physical properties of carrageenan in solution and gel state. Carbohydrate Polym. 1982; 2: 270-272.

7 Gunning A. P. \& Morris V. J. Light scattering studies of tetramethyl ammonium gellan. Int. J. Biol. Macromol. 1990; 12: 338-341.

8 Miyoshi, E. \& Nishinari, K. Rheological and thermal properties near the sol-gel transition of gellan gum aqueous solutions. Progr. Colloid Polym. Sci. 114, 68-82 (1999).

9 Oakenfull, D., Nishinari, K. \& Miyoshi, E. in Hydrocolloids Part 2 (ed. Nishinari K.) 153-163 (Elsevier: Amsterdam, 2000).

10 del Carmen Núñez-Santiago, M. \& Tecante, A. Rheological and calorimetric study of the sol-gel transition of $\kappa$-carrageenan. Carbohydrate Polym. 69, 763-773 (2007).

11 Tanaka, F. Polymer Physics 129-162. (Cambridge University Press: Cambridge, 2011).

12 Stauffer, D., Coniglio, A. \& Adam, M. Gelation and critical phenomena. Adv. Polym. Sci. 44, 103-158 (1982).

13 Takigawa, T., Araki, H., Takahashi, K. \& Masuda, T. Effects of mechanical stress on the volume phase transition poly(N-isoprpylacrylamide) based polymer gels. J. Chem. Phys. 113, 7640-7645 (2000).

14 Takigawa, T., Takahashi, K., Araki, H. \& Masuda, T. Transition enthalpy for a poly(Nisoprpylacrylamide) based polymer gel estimated from the Clapeyron equation. Polym. J. 33, 297-300 (2001) 